(c) 2021 Universidad Nacional Autónoma de México, Facultad de Estudios Superiores Zaragoza.

Este es un artículo Open Access bajo la licencia CC BY-NC-ND (http://creativecommons.org/licenses/by-nc-nd/4.0/).

TIP Revista Especializada en Ciencias Químico-Biológicas, 24: 1-7, 2021.

https://doi.org/10.22201/fesz.23958723e.2021.331

\title{
Comportamiento ecofisiológico de tres cultivares de ejote Phaseolus vulgaris L. (Fabaceae) bajo un clima de transición templado a seco
}

\author{
Humberto Rafael Bravo-Delgado ${ }^{1}$, Mariana Lizbeth Ortega Martínez ${ }^{3 a}$, \\ Juan Manuel Loeza-Corte ${ }^{2}$ y Ernesto Díaz-López ${ }^{3 *}$ \\ ${ }^{1}$ Depto. de Investigación y Desarrollo, CA. Ingeniería en Procesos Alimentarios, Universidad Tecnológica \\ de Tehuacán. ${ }^{3}$ Depto. de Investigación y Desarrollo, CA. Ecofisiología aplicada a cultivos en zonas áridas, \\ Ingeniería en Agricultura Sustentable y Protegida. ${ }^{a}$ Estudiante, Universidad Tecnológica de Tehuacán. \\ Prolongación de la 1 sur \# 1101, San Pablo Tepetzingo Tehuacán 75859, Puebla, México. ${ }^{2}$ Universidad de la \\ Cañada, Teotitlán de Flores Magón 68540, Oaxaca, México. E-mail: *ernesto.lopez@uttehuacan.edu.mx
}

\begin{abstract}
RESUMEN
Con el objeto de conocer el comportamiento de tres cultivares de frijol ejotero, bajo un clima de transición templado a seco y durante el ciclo de cultivo invierno-primavera, se sembraron los genotipos Strike, Valentino y Saporo de crecimiento determinado en Tepanco de López, Puebla a una densidad de 12.5 plantas $\mathrm{m}^{-2}$, con una fertilización de 150-90-60 de NPK. Para conocer la relación cultivo-ambiente, se realizó el estudio del comportamiento ecofisiológico de los genotipos, bajo un diseño de bloques al azar. Los tratamientos fueron los cultivares de frijol ejotero y las variables de respuesta: el número de vainas, rendimiento, color y forma de la vaina, unidades de calor y evapotranspiración. Para tener un mejor control del experimento, se monitorearon las normales climatológicas, temperatura máxima, mínima y precipitación durante el ciclo de cultivo, con ayuda de una estación meteorológica automatizada. Los resultados indican que la evapotranspiración máxima fue de $166.54 \mathrm{~mm}$ alcanzando los tres cultivares la madurez comercial a los $1,192{ }^{\circ} \mathrm{C} \mathrm{día}^{-1}$. El mayor rendimiento de ejote, correspondió al cultivar Strike, por ser el mejor adaptado a las condiciones ecológicas del clima de transición. De este estudio se concluye, que Strike es un genotipo recomendable a los productores de la zona de estudio.

Palabras clave: evapotranspiración, plasticidad fenotípica, rendimiento de ejote, unidades de calor.
\end{abstract}

Ecophysiological behavior of three cultivars of snap bean Phaseolus vulgaris $\mathrm{L}$.

(Fabaceae) under a temperate to dry transitional climate

\begin{abstract}
In order to know the behavior of three cultivars of snap bean, under a temperate to dry transitional climate and during the winter-spring growing cycle, Strike, Valentino and Sapporo genotypes of determined growth were sown in Tepanco de López, Puebla at a density of 12.5 plants $\mathrm{m}^{-2}$, with a fertilization of 150-90-60 of NPK. To know the crop-environment relationship, the study of the ecophysiological behavior of the genotypes was carried out, under a randomized block design. The treatments were the snap bean cultivars and the response variables: pod number, yield, pod color and shape, heat units and evapotranspiration. To have a better control of the experiment, the climatological normals, maximum and minimum temperatures and precipitation were monitored during the cultivation cycle, with the help of an automated meteorological station. The results indicate that the maximum evapotranspiration was $166.54 \mathrm{~mm}$, the three cultivars reaching commercial maturity at $1,192^{\circ} \mathrm{C}_{\text {day }^{-1}}$. The highest snap bean yield corresponded to the Strike cultivar, as it was the best adapted to the ecological conditions of the transitional climate. From this study it is concluded that Strike is a recommended genotype for producers in the study area.
\end{abstract}

Keywords: evapotranspiration, phenotypic plasticity, snap bean yield, heat units.

Artículo recibido el 07 de septiembre del 2020.

Artículo aceptado el 26 de junio del 2021. 


\section{INTRODUCCIÓN}

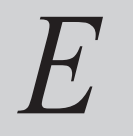

1 frijol (Phaseolus vulgaris L.) es un cultivo básico, domesticado en Mesoamérica por las culturas precolombinas (Miranda, 1967) citado por (Hernández, Vargas, Maruaga, Hernandéz \& Mayek, 2013). Pertenece a la familia Fabaceae y con la capacidad de fijar nitrógeno atmosférico, debido a la asociación simbiótica con bacterias del género Rhizobium, al proceso se le conoce como fijación biológica del nitrógeno (Ángeles \& Cruz, 2015; Mayz, 2004). El frijol en México, cuando es destinado al consumo humano como una hortaliza, se llama ejote, judía verde en España y poroto en Sudamérica (Ulloa, Rosas, Ramírez \& Ulloa, 2011). Su consumo en fresco, aporta a la dieta del ser humano, vitaminas como: retinol, vitamina B6 y K, además de ser una fuente importante de $\mathrm{Ca}^{++}, \mathrm{Fe}^{++}, \mathrm{K}^{+}$y fibra (Salinas, Escalante, Rodríguez \& Sosa, 2012). Lo anterior ha propiciado que el cultivo de esta leguminosa se incremente en México, ocupando el cuarto lugar de importancia en cuanto a la superficie sembrada, después del maíz, pastos y sorgo (FIRA, 2016). En México los principales estados productores son: Morelos, Puebla, Hidalgo y Sinaloa que producen el 78.9\% a nivel nacional (SAGARPA, 2009). Este cultivo es adaptable a múltiples climas, pero es susceptible a la conductividad eléctrica del suelo y cuando ésta es superior a $3.00 \mathrm{dS} \mathrm{m}^{-1}$ se reduce el rendimiento en un 50\% (Quintana, Blanco, Pinzón, Sandoval \& Fernando Torres, 2016). En la zona de transición de clima templado a seco del estado de Puebla, el agua de riego contiene elevados niveles de carbonatos considerándose como (aguas duras). Bajo esta tendencia Salinas, Escalante, Rodríguez \& Sosa (2013), mencionan que los cultivares de ejote utilizados en zonas de suelos carbonatados, no están bien adaptados y tienen bajos rendimientos de 3.7 ton $\mathrm{ha}^{-1}$ que no satisfacen el consumo per cápita. Por su parte Roy, Laflame \& Tremblay (2000), aseveran que otro factor de importancia que contribuye al bajo rendimiento del ejote, es la escasa y heterogeneidad manifiesta en la distribución de la precipitación, cuando se practica agricultura de temporal. Lo anterior, ha sido comprobado por Mayor, Bodo \& Blair (2016), quienes señalan que el frijol es una especie cosmopolita, sin embargo, el $60 \%$ de los cultivos de frijol corren el riesgo de una sequía intermitente o terminal, por ello se deben idear estrategias para contrarrestar los efectos de la misma. Otro factor son las altas tasas de radiación que provocan una mayor evaporación y ocasionan la alcalinización del suelo. Para el cultivo exitoso del frijol ejotero, es necesario crear cultivares resistentes a la salinidad del suelo y el $\mathrm{pH}$ alcalino (Morales, López, Kohashi, Miranda \& García, 2015; Durán, Lambert \& Velázquez, 2014), además de evaluar algunos aspectos ecofisiológicos que permitan conocer la adaptabilidad de los cultivares disponibles para una determinada región. Por ello, el objetivo principal de esta investigación consistió en estudiar ecofisiológicamente el comportamiento de tres cultivares de frijol ejotero, en un clima de transición templado a seco, en el estado de Puebla, México. La hipótesis planteada fue si la respuesta de los cultivares en estudio es diferente cuando son sembrados bajo un clima de transición templado a seco, debido a la plasticidad fenotípica de cada uno de ellos.

\section{MATERIALES Y MÉTODOS}

\section{Ubicación del experimento y clima}

La presente investigación se llevó a cabo en Tepanco de López, Puebla, México, ubicado a $18^{\circ} 33^{\prime} 18^{\prime \prime}$ latitud norte, $97^{\circ} 33^{\prime \prime}$ $37^{\prime \prime}$ longitud oeste y 1,805 metros de altitud. Bajo un clima $\mathbf{C}(\mathbf{b})\left(\mathbf{h}^{\prime}\right)\left(\mathbf{w}^{\prime}\right) \mathbf{i}^{\prime} \mathbf{g}$, que corresponde al de transición templado a seco, con temperatura media anual de $18^{\circ} \mathrm{C}$, y precipitación total de $464 \mathrm{~mm}$, cuyo régimen de lluvias va de mayo a septiembre, presencia de sequía intraestival, oscilación de la temperatura entre el mes más cálido y frío entre $5{ }^{\circ} \mathrm{C}$ y $7{ }^{\circ} \mathrm{C}$, el mes más cálido se presenta antes del solsticio de verano, siendo el mes de mayo como se indica para esta zona (García, 2005). Figura 1.

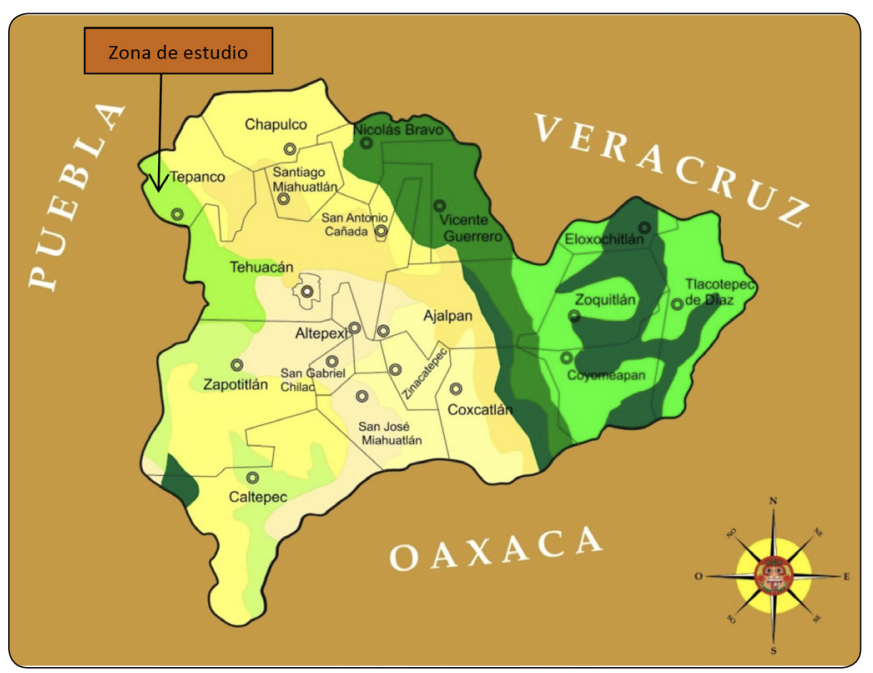

Figura 1. Principales climas y subclimas de Tehuacán y sus principales regiones, Tehuacán, Puebla. Año 2020. (Tomado de: Academia Tlatoani, 2020).

\section{Cultivares, arreglo topológico y unidad experimental}

Para el experimento se seleccionaron tres cultivares de frijol ejotero: "Strike", "Valentino" y "Saporo"; que corresponden a cultivares de ejote claro, vainas rectas, aterciopeladas a los 52 días de su madurez y resistencia al virus 1 del frijol (BV1), todos de crecimiento determinado y tolerantes a suelos con alta conductividad eléctrica. La siembra se hizo el 2 de enero del año 2018, en un suelo vertisol en proceso de formación, bajo una densidad de 12.5 plantas $\mathrm{m}^{-2}$. La unidad experimental se constituyó de 5 surcos, cada uno de 5 metros de longitud, separados $0.80 \mathrm{~m}$, siendo la parcela útil el surco central, y la unidad experimental una planta.

\section{Fertilización y diseño experimental}

La fertilización fue de 150-90-60 de NPK, utilizando como fuente de nutrimentos la urea de $(46 \% \mathrm{~N})$, superfosfato de calcio triple de $\left(46 \% \mathrm{P}_{2} \mathrm{O}_{5}\right)$ y cloruro el de potasio $\left(\mathrm{K}_{2} \mathrm{O}\right)(60 \%)$, 
aplicados al momento de la siembra. El diseño experimental utilizado consistió en, bloques completos al azar $(3 \times 5)=15$ unidades experimentales, evaluado bajo el modelo matemático $\mathbf{Y}_{\mathrm{ij}}=\boldsymbol{\mu}+\mathbf{T}_{\mathbf{i}}+\boldsymbol{\beta}_{\mathbf{j}}+\boldsymbol{\varepsilon}_{\mathrm{ij}}$, donde: $\mathrm{Y}_{\mathrm{ij}}$, es la variable respuesta del i-ésimo cultivar de frijol ejotero en el j-ésimo bloque; $\mu$, es la media general verdadera; $\mathrm{T}_{\mathrm{i}}$, es el efecto del i-ésimo cultivar; $\beta_{\mathrm{j}}$, es el efecto del j-ésimo bloque o repetición y $\varepsilon_{\mathrm{ij}}$, es el error experimental del i-ésimo cultivar en el j-ésimo bloque (Cochran \& Cox, 2008; Steel \& Torrie, 1996).

\section{Variables de respuesta}

Las variables de respuesta fueron: altura de la planta, medida desde el inicio del epicótilo hasta la yema apical; área foliar total, utilizando un vernier digital de 8" y la fórmula para el área del triángulo $\mathbf{A F}=$ dónde: $\mathbf{A F}$, es el área foliar total; $\mathbf{B}$, la base y h, la altura del foliolulo; el rendimiento agronómico es el resultado del peso total de vainas de ejote y cuando su longitud fue mayor a $3 \mathrm{~cm}$, se pesaron en una balanza digital modelo BAPRE-3 de $3 \mathrm{~kg}$ de capacidad; contando también el número total de vainas por planta; se utilizó un vernier digital para medir la longitud de las vainas desde la base hasta el ápice y su ancho a partir del centro. Se usó la tabla de color Munsell para tejidos vegetales con la finalidad de identificar el color de las vainas y la forma, tomando como referencia la guía técnica para la descripción varietal del (SNICS, 2017). Las unidades de calor, se evaluaron por el método residual de Snyder (1985) y la ecuación $\mathbf{U C}=$ donde; $\mathrm{UC}$, son las unidades de calor $\left({ }^{\circ} \mathrm{C}\right.$ día $\left.{ }^{-1}\right)$; Tmáx y Tmín, temperaturas máxima y mínima respectivamente y $\mathrm{Tb}$, es la temperatura base del cultivo, en este caso para el frijol ejotero es de $10^{\circ} \mathrm{C}$ (Escalante \& Rodríguez, 2001). Para medir la evapotranspiración, la fórmula es $\mathbf{E T c}=\mathbf{E V} * \mathbf{K c} * \mathbf{K e}$, donde ETc, es la evapotranspiración del cultivo en mm; EV, es la evaporación medida con el evaporímetro de tanque tipo "A"; Kc, es el coeficiente del cultivo 0.65 y Ke, es el coeficiente del evaporímetro 0.60 (Doorenbos \& Pruitt, 1986), citado por (Olalde, Escalante, Sánchez, Tijerina, Ebgleman \& Mastache, 2000). A las variables significativas, se les aplicó la prueba de comparación múltiple de Tukey, a un nivel de significancia del $5 \%$ de probabilidad de error.

\section{RESULTADOS Y DISCUSIÓN \\ Diagrama ombrotérmico}

En la Figura 2 se presenta la dinámica de la temperatura máxima y mínima del frijol ejotero, durante su ciclo de cultivo invierno-primavera. En la que se aprecia, una temperatura máxima entre $23.6^{\circ} \mathrm{C}$ y $28^{\circ} \mathrm{C}$, y una temperatura mínima entre $2.2^{\circ} \mathrm{C}$ y $9.5^{\circ} \mathrm{C}$. Respecto a la precipitación, la máxima fue de $76.9 \mathrm{~mm}$ en el mes de mayo, con un promedio de precipitación del $52.77 \%$ durante el ciclo del cultivo y un $47.23 \%$ para el periodo enero-abril. Es importante mencionar, que bajo estas condiciones ecológicas, el cultivo alcanzó la etapa fenológica de $\mathrm{R}_{4}$ (Apáez, Escalante, Sosa, Rodríguez \& Apáez, 2014), correspondiente al corte comercial de la vaina, de un cultivo que se desarrolló adecuadamente.

\section{Variables de respuesta}

Respecto a las variables de respuesta en la Tabla I, se presenta el análisis de varianza y la prueba de comparación múltiple para seis variables. El análisis de varianza, mostró diferencias altamente significativas para la altura de la planta, el área

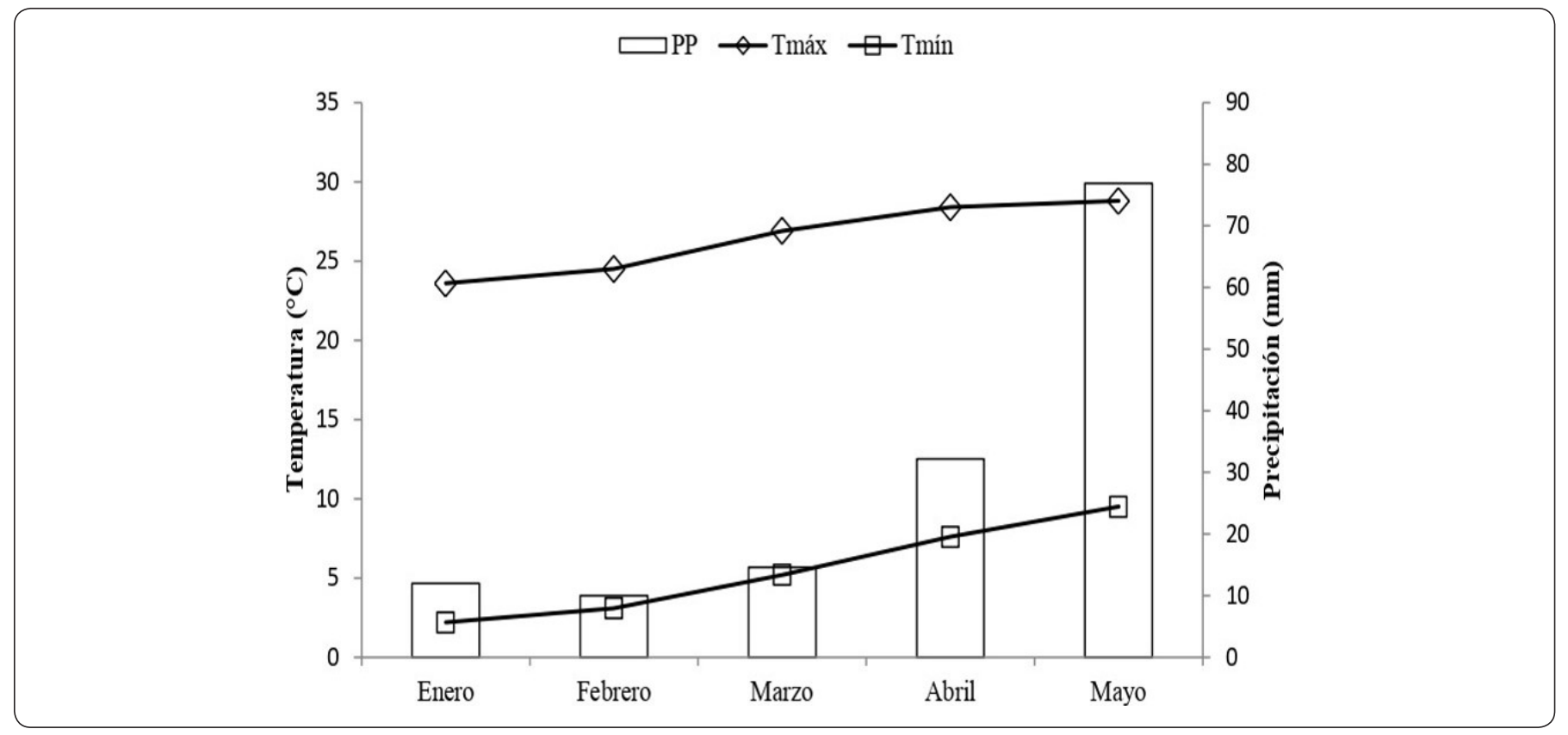

Figura 2. Temperatura máxima, mínima y precipitación mensual, en Tepanco de López, Puebla. Ciclo invierno-primavera del año 2018. Tmáx, temperatura máxima; Tmín, temperatura mínima; PP, precipitación. 
Tabla I. Análisis de varianza y prueba de comparación múltiple, para seis variables de respuesta de tres cultivares de frijol ejotero (Phaseolus vulgaris L.). Tepanco de López, Puebla. Año 2018.

\begin{tabular}{|l|c|c|c|c|c|c|}
\hline \multicolumn{1}{|c|}{ Cultivares } & $\begin{array}{c}\mathbf{A P} \\
\mathbf{c m}\end{array}$ & $\begin{array}{c}\mathbf{A F} \\
\mathbf{c m}^{\mathbf{2}}\end{array}$ & $\begin{array}{c}\mathbf{R} \\
\mathbf{g} \text { planta }\end{array}$ & $\mathbf{N V}$ & $\begin{array}{c}\mathbf{A V} \\
\mathbf{c m}\end{array}$ & $\begin{array}{c}\mathbf{L V} \\
\mathbf{c m}\end{array}$ \\
\hline Strike & $38.50 \mathrm{~b}$ & $2302.80 \mathrm{a}$ & $82.57 \mathrm{a}$ & $15.33 \mathrm{a}$ & $0.684 \mathrm{a}$ & $13.07 \mathrm{a}^{*}$ \\
\hline Valentino & $33.50 \mathrm{c}$ & $938.10 \mathrm{~b}$ & $54.57 \mathrm{~b}$ & $13.33 \mathrm{a}$ & $0.691 \mathrm{a}$ & $11.19 \mathrm{~b}$ \\
\hline Saporo & $45.00 \mathrm{a}$ & $2585.25 \mathrm{a}$ & $38.37 \mathrm{~b}$ & $14.00 \mathrm{a}$ & $0.554 \mathrm{a}$ & $13.07 \mathrm{a}$ \\
\hline DSH & $4.60 * *$ & $677.33^{* *}$ & $22.46^{* *}$ & $4.68^{\text {n.s }}$ & $0.273^{\text {n.s }}$ & $1.06^{* *}$ \\
\hline CV\% & 10.33 & 25.73 & 28.10 & 4.44 & 5.79 & 20.32 \\
\hline
\end{tabular}

*Medias dentro de columnas con la misma literal, estadísticamente son iguales según Tukey a $(p \leq 0.05)$. AP, altura de la planta; AF, área foliar; R, rendimiento; NV, número de vainas; $\mathrm{AN}$, ancho de la vaina; LV, longitud de la vaina; DSH, diferencia significativa honesta; CV, coeficiente de variación; **; n.s, significativo al 0.01 y no significativo.

foliar, el rendimiento y la longitud de vaina. A pesar de existir diferencias numéricas para el número y ancho de la vaina, estadísticamente resultaron iguales. El coeficiente de variación indicó, que los datos son confiables para la altura de la planta, el número y ancho de la vaina, siendo este menor al $15 \%$, mientras que para el área foliar, el rendimiento y la longitud de la vaina, los datos como en el caso anterior son fidedignos, ya que el coeficiente de variabilidad (CV), fue mayor al $15 \%$ y menor al 30\% respectivamente (Steel \& Torrie, 1996). En relación a la prueba de comparación múltiple, los cultivares Strike y Saporo, presentaron un mayor despliegue del área foliar con $2,302.80$ y $2,585.25 \mathrm{~cm}^{2}$, superando al genotipo Valentino, que fue de $938.10 \mathrm{~cm}^{2}$ y la longitud de la vaina $11.19 \mathrm{~cm}$, media rebasada por Strike y Saporo. Respecto al rendimiento de la vaina, en Strike fue mayor con $82.57 \mathrm{~g} \mathrm{planta}^{-1}$, más que en los de Valentino y Saporo, con resultados estadísticamente iguales con 54.57 y 38.37 g planta $^{-1}$ respectivamente.

Lo anterior se atribuye, a que Strike y Saporo mostraron una mayor área foliar, lo que permitió además de un aumento en la captación de la radiación solar, un aumento en la incorporación de los carbohidratos a la vaina, circunstancias que favorecieron el rendimiento agronómico. Lo reportado en esta investigación, no concuerda con lo obtenido por Acosta, Amador \& Padilla (2008), quienes mencionan que entre el índice del área foliar (IAF), que es otra forma de evaluar el área foliar, presenta una baja correlación con el rendimiento. Por su parte Aguilar, Escalante, Ficikovsky, Tijerina \& Mark (2005), al trabajar con girasol mencionan que el rendimiento agronómico, presenta una correlación alta y positiva con la duración del área foliar.

A este respecto Aguilar, Escalante \& Aguilar (2015), refieren que un cultivo de maíz cultivar H-562 bajo las condiciones climatológicas de Iguala, Guerrero, con la aplicación de $160 \mathrm{~kg} \mathrm{ha}^{-1}$ de N, aumentó el tamaño y la duración del aparato fotosintético, es decir tanto el índice como la duración del área foliar, repercutiendo en el incremento de la tasa de crecimiento
Tabla II. Color de la vaina de frijol ejotero (Phaseolus vulgaris L.) y forma del fruto, de tres variedades de ejote. Tepanco de López, Puebla. Año 2018.

\begin{tabular}{|l|l|l|}
\hline \multicolumn{1}{|c|}{ Cultivares } & \multicolumn{1}{c|}{ Color } & \multicolumn{1}{c|}{ Forma } \\
\hline Strike & $7.5 \mathrm{GY} 8 / 4$ & Curvada \\
\hline Valentino & $7.5 \mathrm{GY} 6 / 6$ & Curvada \\
\hline Saporo & $7.5 \mathrm{GY} 7 / 6$ & Curvada \\
\hline
\end{tabular}

del cultivo y la tasa de asimilación neta y per se, incrementan el rendimiento.

Respecto al color y forma de la vaina, de los tres cultivares presentaron un matiz 7.5 GY (verde-amarillo), mientras que Strike presentó el valor más alto en (Value) 8 , seguido de Saporo con 7 y Valentino con 6; de este modo el croma más alto fue para Valentino y Saporo con 6, mientras que Strike alcanzó solo 4 puntos (ver Tabla II). Respecto a la forma, todos los cultivares fueron curvados, lo que coincide con la descripción cóncava y verde del grupo flor de Mayo M-38 descrita por SNICS, (2017). De igual forma Esquivel., Acosta, Rosales, Pérez, Hernández, Navarrete \& Muruaga (2006) mencionan que el ejote debe tener poca fibra, mesocarpo grueso y suculento y forma alargada, para que se considere de calidad como ocurrió en el presente estudio.

En la Figura 3, se presentan las unidades de calor acumuladas para tres cultivares de frijol ejotero. En ella se aprecia que se acumularon 1,192 unidades de calor, desde la siembra hasta el último corte de la vaina. El modelo de ajuste fue lineal, con un coeficiente de determinación alto, indicativo de que el $96 \%$ de las unidades de calor son explicadas por los días después de la siembra. Por lo que la pendiente del modelo 1.76 indica, que por cada día que pasa, el cultivo acumula 1.76 unidades de calor. Lo reportado en este estudio, difiere con los datos de Delgado, Escalante, Morales, López \& Rocandio (2015), quienes reportan en frijol una acumulación de $950^{\circ} \mathrm{C}$ 


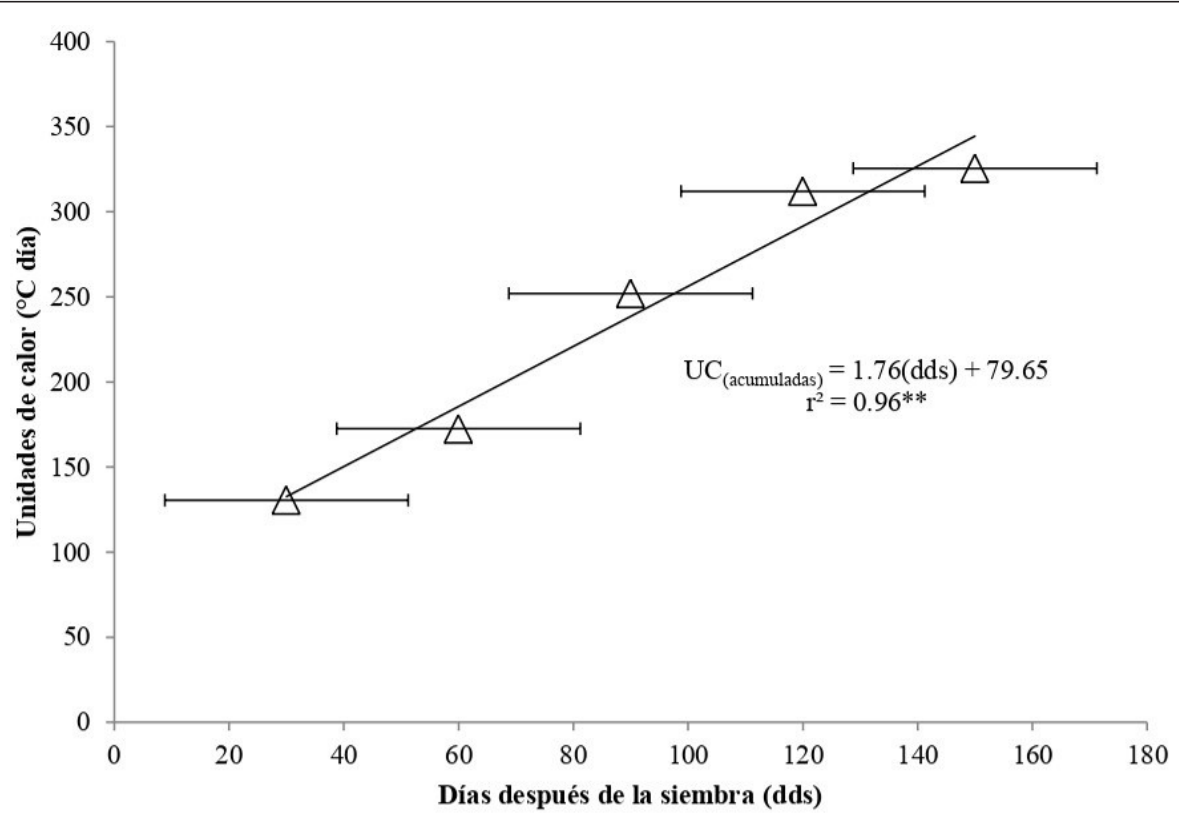

Figura 3. Unidades de calor acumuladas, para tres cultivares de frijol ejotero (Phaseolus vulgaris L.), en Tepanco de López, Puebla. Ciclo invierno-primavera, 2018. UC, unidades de calor; dds, días después de la siembra. **; significativo al 0.01.

día, es decir 20.4\% menos. Por su parte Díaz, Escalante, Rodríguez \& Gaytán (2010), reportan una acumulación de 1,319 unidades de calor bajo un clima seco, 9.7\% más de unidades de calor acumuladas. Con esta integral térmica, el cultivo completó su ciclo ontogénico en el clima de la zona, por ello los genotipos se pueden recomendar para ser sembrados en la región.

En la Figura 4 se presenta la evapotranspiración del cultivo. La gráfica se ajustó a un modelo cuadrático, con coeficiente de determinación alto y significativo 0.90. A los $90 \mathrm{dds}$, el $52.55 \%$ de la evapotranspiración que corresponde a $166.54 \mathrm{~mm}$, ocurrieron en este periodo, alcanzando su máximo a los $120 \mathrm{dds}$, para posteriormente disminuir. Este hecho se explica por la nubosidad, ocurrida durante el mes de mayo, lo que provocó que la radiación solar, no llegara con mayor intensidad al suelo, disminuyendo así la evapotranspiración. Este efecto ha sido corroborado por Sánchez \& Carvacho (2011), quienes mencionan que la nubosidad, afecta la medición de la radiación solar, parámetro que es de suma importancia, para medir la evapotranspiración, cuando se utiliza el método de Turc. El modelo matemático, muestra que entre los 90 y 120 días después de la siembra, el cultivo presentó la mayor evapotranspiración, por ello es necesario tomar en cuenta este dato, ya que en este intervalo la planta está en plena producción de ejote y la humedad no debe de faltar, para obtener una buena producción al lograr una mayor eficiencia en el uso del agua, por efecto de la evapotranspiración, como lo mencionan Martínez, Jasso \& Huerta (2012).

\section{Conclusiones}

Los cultivares en estudio se adaptaron a las condiciones de un clima de transición, con altos rendimientos, comparados con los de las zonas estrictamente ejoteras. Destacando la gran plasticidad fenotípica del cultivar Strike. El cultivo de frijol ejotero, puede ser una alternativa real para los productores de regiones, con altas tasas de evapotranspiración. El resultado del comportamiento ecofisiológico de los cultivares de este estudio, puede ser un ejemplo útil para la búsqueda de otros genotipos que tengan un rendimiento aceptable para ser cultivados.

\section{REFERENCIAS}

Academia Tlatoani. (2020). Los clomas de Tehuacán. Disponible en: http://academiatlatoani.blogspot. com/2013/01/mapas-de-tehuacan.html

Acosta, D. E., Acosta, G. J. A., Amador, R. M. D. \& Padilla, R. J. S. (2008). Relationship between the leaf area index and yield of dry bean grown under rainfed conditions. Agricultura técnica en México, 34(1), 1320. Obtenido el 10 de Marzo de 2020, de: http://www. scielo.org.mx/scielo.php?script=sci_arttext\&pid=S056825172008000100002\&lng=en\&tlng=.

Aguilar, C. C., Escalante, E. J. A. \& Aguilar, M. I. (2015). Análisis de crecimiento y rendimiento de maíz en clima cálido en función del genotipo, biofertilizante y nitrógeno. Terra Latinoamericana, 33(1), 51-62. Recuperado en 24 de Abril de 2020, de http://www. scielo.org.mx/scielo.php?script=sci_arttext\&pid=S018757792015000100051\&lng=es\&tlng=es. 


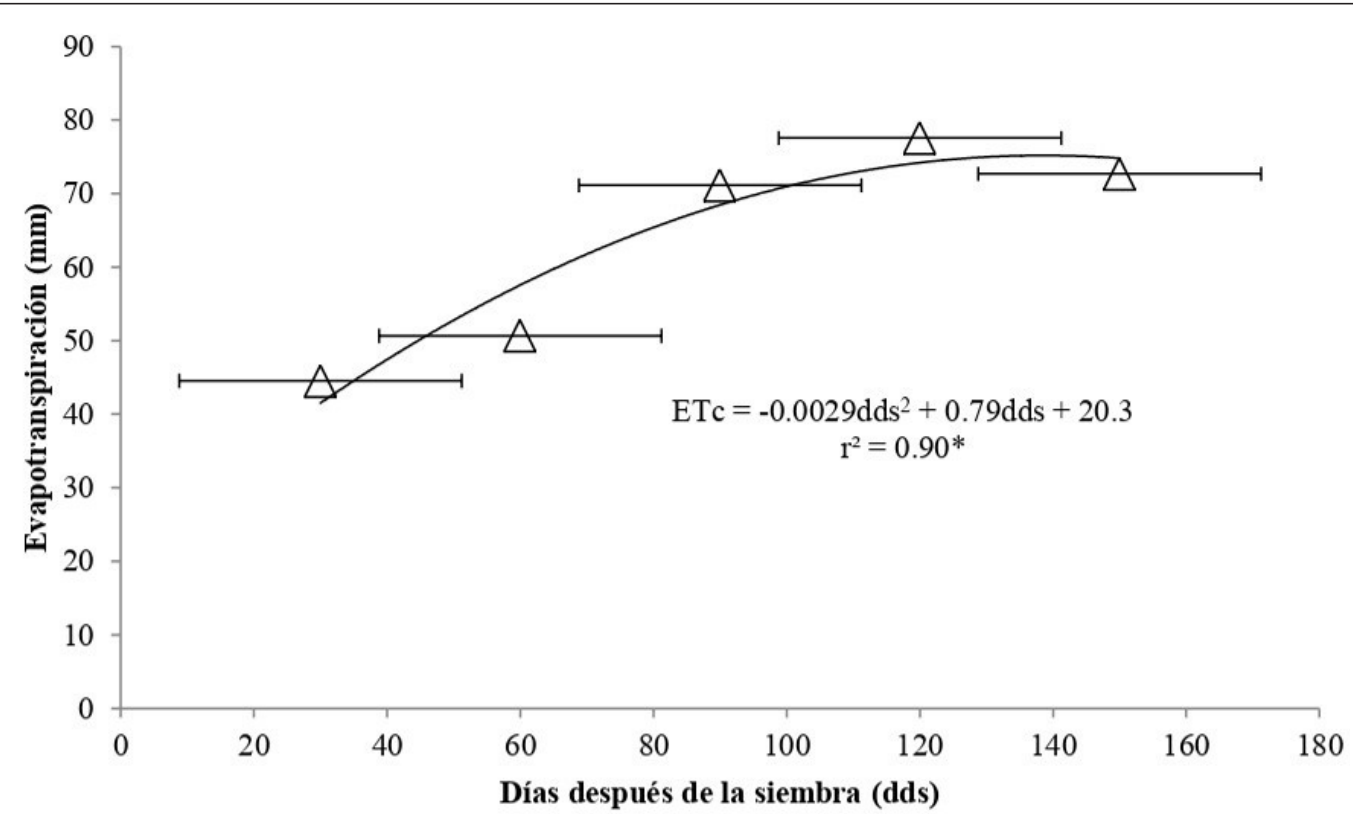

Figura 4. Evapotranspiración de tres cultivares de ejote (Phaseolus vulgaris L.), en un clima de transición templado-seco, en Tepanco de López, Puebla. Ciclo de cultivo invierno-primavera, 2018. ETc, evapotranspiración; dds, días después de la siembra. *; significativo al 0.05

Aguilar, G. L., Escalante, E. J. A., Fucikovsky, Z. L., Tijerina, Ch. L. \& Mark, E. E. (2005). Área foliar, tasa de asimilación neta, rendimiento y densidad de población en girasol. Terra Latinoamericana, 23(3), 303-310. Recuperado el 1 de Agosto de 2020, De: https://www.redalyc.org/articulo. oa?id $=573 / 57311101001$.

Ángeles, N. J. G. \& Cruz, A. T. (2015). Isolation, molecular characterization and evaluation of nitrogen-fixing strains in promoting the growth ofbeans. RevistaMexicanade Ciencias Agrícolas, 6(5), 929-942. Recuperado el 18 de mayo de 2020, de http://www.scielo.org.mx/scielo.php?script=sci arttext\&pid=S2007-09342015000500002\&lng=en\&tlng=.

Apáez, B. P., Escalante, E. J. A. S., Sosa, M. E., Rodríguez, G. M. T. \& Apáez, B. M. (2014). Fenología, producción y calidad nutrimental del grano de frijol chino en función de la biofertilización y fertilización foliar. Interciencia, 39(12), 857-862. https://www.redalyc.org/ pdf/339/33932786005.pdf

Cochran, G. W. \& Cox, M. G. (2008). Diseños Experimentales. Trillas. $2^{\mathrm{a}}$ Ed. México, D. F. 661 p.

Delgado, M. R., Escalante, E. J. A. S., Morales, R. E. J., López, S. J. A. \& Rocandio, R. M. (2015). Snap bean (Phaseolus vulgaris L.) production and profitability intercropping with maize in function of density and nitrogen in template climate. Rev. FCA UNCUYO, 47(2), 15-25. Recuperado el 02 de agosto de 2020, de www.289996556_Snap_bean_ Phaseolus_vulgaris_L_production_and_profitability_ intercropping_with_maize_in_function_of_density_and_ nitrogen_in_template_climate.
Díaz, L. E. Escalante, E. J.A., Rodríguez, G. M. T. \& Gaytán,A. E. A. (2010). Producción de frijol ejotero en función del tipo de espaldera. Revista Chapingo Serie Horticultura, 16(3), 215221. Recuperado el 04 de junio de 2020, de de http://www. scielo.org.mx/scielo.php?script=sci_arttext\&pid=S1027152X2010000300010\&lng=pt\&tlng=es.

Doorenbos, J. \& Pruitt, D.O. (1986). Crop water requirements. Irrig. and Drain. Paper 24. Food and Agric. Organization of the United Nations. Rome, Italy.

Durán, A. A., Lambert, G. T. \& Velázquez, F. R. (2014). Evaluation of improved genotypes of black bean Phaseolus vulgaris L. in Barinas and Monagas, Venezuela. Revista de Ciencias Agrícolas, 31(2), 63-75. DOI: http://dx.doi. org/10.22267/rcia.143102.32

Escalante, E. J. A., Escalante, E. L. E. \& Rodríguez, G. M. T. (2001). Bean Production in Two Planting Seasons and Their Relationship with Evapotranspiration, Heat Units and Solar Radiation in Hot Climate. Terra Latinoamericana, 19(4), 309-315. Recuperado en: 24 de Agosto de 2020]. ISSN: . Disponible en 12 e abril de 2020, en: https:/www.redalyc. org/articulo.oa?id=573/57319403

Esquivel, E. G., Acosta, G. J. A., Rosales, S. R., Pérez, H. P., Hernández, C. J. M., Navarrete, M. R. \& Muruaga, M. J. S. (2006). Productividad y adaptación del frijol ejotero en el Valle de México. Revista Chapingo Serie Horticultura, 12(1), 119-126. https:/www.redalyc.org/ pdf/609/60912116.pdf

Fideicomisos Instituidos en Relación con la Agricultura. (2016). Panorama Agroalimentario "Frijol”. Dirección 
de Investigación y Evaluación Económica y Sectorial. 36 p. Recuperado en 18 de marzo de 2020, en: https:/www. gob.mx/cms/uploads/attachment/file/200638/Panorama_ Agroalimentario_Frijol 2016.pdf

García, E. (2005). Modificación al Sistema de Clasificación Climática de Köppen. 4a ed. UNAM. México, D.F. 217 p.

Guía técnica para la descripción varietal de frijol. (2017). Servicio Nacional de Inspección y Certificación de Semillas. Secretaria de Agricultura, Ganadería, Desarrollo Rural, Pesca y Alimentación. 38 p.

Hernández, L. V, M., Vargas, V. L. P., Maruaga, M. J. S., Hernández, D. S. \& Mayek, P. N. (2013). Origin, domestication and diversification of common beans. advances and perspectives. Revista Fitotecnia Mexicana, 36(2), 95-104. Recuperado en 12 de agosto de 2020, en: https://www.researchgate.net/publication/287466521_ Origin_domestication_and_diversification_of_common beans_advances_and_perspectives

Martínez, G. M. A., Jasso, Ch. C. \& Huerta, D. J. (2012). Effect of fertilization doses with fertigation and conservation tillage in bean yield and soil properties. Revista Mexicana de Ciencias Agrícolas, 3(8), 1551-1564.http://www.scielo. org.mx/pdf/remexca/v3n8/v3n8a6.pdf

Mayor, D. V. M., Bodo, R. \& Blair, M. W. (2016). Desarrollo de líneas de frijol (Phaseolus vulgaris L.) tolerante a sequía a partir de cruces inter acervo con genotipos procedentes de diferentes orígenes (Mesoamericano y Andino). Acta Agronómica, 65(4), 431-437. doi: http:// dx.doi.org/10.15446/acag.v65n4.48680

Mayz, F. J. (2004). Biological nitrogen fixation. Revista UDO Agrícola, 4(1), 1-20. Recuperado en en 10 de agosto de 2020, en: https://dialnet.unirioja.es/descarga/ articulo/2221548.pdf

Miranda, C. S. (1967). Origen de Phaseolus vulgaris L. (Frijol común). Agrociencia, 1(1), 99-104. Recuperado en 15 de agosto de 2020, en: http://www.biblio.colpos.mx:8080/ xmlui/bitstream/handle/10521/1915/Agrociencia\%20 v1n2_1967_99-109.pdf?sequence $=1$

Morales, R. A., López, C. C., Kohashi, S. J., Miranda, C. S. \& García, E. A. (2015). Comparison of Yield Components in Bean Varieties Grown Under Restrictions of Soil of Acidity and Residual Soil Moisture in Southern Veracruz. Terra Latinoamericana, 33(4), 309-319. Recuperado el 10 de agosto de 2020, en https://www. researchgate.net/publication/317437611_Comparison_ of_yield_components_in_bean_varieties_grown_under_ restrictions_of_soil_of_acidity_and_residual_soil_ moisture_in_southern_Veracruz

Olalde, G. V. M., Escalante, E. J. A., Sánchez, G. P., Tijerina, Ch. L., Engleman, C. E, M. \& Mastache, L. A. A. (2000). Eficiencia en el uso del agua y del nitrógeno, y rendimiento del girasol, en función del nitrógeno y densidad de población en clima cálido. Terra, 18(1), 51-59. Recuperado en 10 de agosto de 2020, en https://www.redalyc.org/articulo. oa? $\mathrm{id}=573 / 57318106$

Quintana Blanco, W. A., Pinzón Sandoval, E. H. \& Fernando Torres, D. (2016). Evaluación del crecimiento de fríjol (Phaseolus vulgaris L.) cv. Ica Cerinza, bajo estrés salino. Revista U.D.C.A Actualidad \& Divulgación Cientifica, 19(1), 87-95. DOI: https://doi.org/10.31910/ rudca.v19.n1.2016.113

Roy, G., Laflame, L. \& Tremblay, N. (2000). Évolution des calibres et des rendements de cultivars de haricot destinés à la transformation. Canadian Journal of Plant Science, 80(4), 869-873. DOI: 10.4141/P99-137.

SAGARPA, Secretaría de Agricultura, Ganadería, Desarrollo Rural, Pesca y Alimentación. (2009). Producción de Hortalizas. Disponible en: http://www.sagarpa.gob.com. $\mathrm{mx}$ (Diciembre 2020).

Salinas, R. N., Escalante, E. J. A., Rodríguez, G. M. T. \& Sosa, M. E. (2012). Yield and nutritional quality of snap bean in two environments. Revista Fitotecnia Mexicana, 35(4), 317-323. Recuperado en 12 de julio de 2020, en https://www.researchgate.net/publication/286740983 Yield_and_nutritional_quality_of_snap_bean_in_two_ environments

Salinas, R. N., Escalante, E. J. A. S., Rodríguez, G. M. T. \& Sosa, M. E. (2013). Rendimiento, calidad nutrimental y rentabilidad del frijol ejotero de temporal en San Pablo Ixayoc, México. Revista Chapingo Serie Horticultura, 19(3), 333-342. https://www.redalyc.org/ pdf/609/60929307006.pdf

Sánchez, M. M. \& Carvacho, B. L. (2011). Comparación de ecuaciones empíricas para el cálculo de la evapotranspiración de referencia en la Región del Libertador General Bernardo O'Higgins, Chile. Revista de Geografia Norte Grande, 50(1), 171-186. DOI: http://dx.doi.org/10.4067/S071834022011000300010

Servicio Nacional de Inspección y Certificación de Semillas. (2017). Guía técnica para la descripción varietal. $2^{\mathrm{a}} \mathrm{Ed}$. Secretaría de Agricultura, Ganadería, Desarrollo Rural, Pesca y Alimentación. 27 p. Recuperado en 29 de Julio de 2020, en: https://inehrm.gob.mx/recursos/Libros/ SAGARPA.pdf

Snyder, R. L. (1985). Hand calculating degree days. Agric. For. Meteorol., 35, 353-358. DOI: https://doi.org/10.1016/01681923(85)90095-4

Steel, G. D. R. \& Torrie, H. J. (1996). Bioestadística: Principios

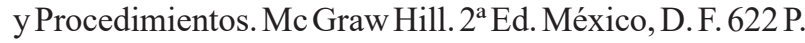

Ulloa, J. A., Rosas, U. P., Ramírez, R. J. C. \& Ulloa, R. B. E. (2011). El frijol (Phaseolus vulgaris L.): su importancia nutricional y como fuente de fitoquímicos. Revista Fuente, 3(8), 5-9. Recuperado en 14 de agosto de 2020, en http:// dspace.uan.mx:8080/jspui/handle/123456789/582 